\title{
Kinetic Sunyaev-Zeldovich effect from galaxy cluster rotation
}

\author{
J. Chluba ${ }^{1,3}$ and K. Mannheim ${ }^{2,3}$ \\ 1 Max-Planck-Institut für Astrophysik, Karl-Schwarzschild-Str. 1, 85740 Garching bei München, Germany \\ e-mail: jchluba@mpa-garching.mpg.de \\ 2 Institut für Theoretische Physik und Astrophysik, Am Hubland, 97074 Würzburg, Germany \\ e-mail: mannheim@astro.uni-wuerzburg.de \\ 3 Universitäts-Sternwarte Göttingen, Geismarlandstr. 11, 37083 Göttingen, Germany \\ Received 12 August 2002 / Accepted 25 September 2002
}

Abstract. We show how the temperature and the polarization of the cosmic microwave background are affected by bulk rotation of clusters of galaxies owing to the kinetic Sunyaev-Zeldovich effect. The main effects of rotation are (i) a shift of the position of the peak of the temperature fluctuation relative to the center of the cluster by a few percent of the core radius and (ii) a tilt of the direction of the plane of linear polarization by several degrees.

Key words. cosmology: cosmic microwave background - cosmology: miscellaneous

\section{Introduction}

Several effects lead to anisotropies of the cosmic microwave background $(\mathrm{CMB})$ : primary effects, imprinted on the surface of last scattering, and secondary effects, arising after hydrogenrecombination or after reionization has taken place (for references see White \& Cohn 2002).

Among the secondary effects, the thermal SunyaevZeldovich effect (th-SZE), which is due to inverse Compton scattering of the CMB photons off the hot intracluster medium (ICM) (Zeldovich \& Sunyaev 1969), and the kinetic SunyaevZeldovich effect (k-SZE), which arises from the peculiar motion of the cluster in the rest frame of the CMB (Sunyaev \& Zeldovich 1980), are most important. The cosmological importance of the th-SZE is due to the fact that it is redshift independent. Therefore it can be used to detect clusters of galaxies at redshifts, where other observational methods fail (Korolev et al. 1986; Kneissl et al. 2001), and to determine the Hubble constant (Birkinshaw 1999; Reese et al. 2002). In principle the $\mathrm{k}-\mathrm{SZE}$ can be used to determine both components of the peculiar velocity: the line of sight component via the relative change of intensity of the $\mathrm{CMB}$ and the velocity component in the celestial plane via the degree of linear polarization of the CMB radiation. Measurements of the k-SZE are extremely difficult but will probably become feasible in the near future.

In this paper we address the k-SZE arising from bulk rotation of the ICM. In order to distinguish this effect from the $\mathrm{k}-\mathrm{SZE}$ due to a bulk translatory motion of the cluster with respect to the CMB, we shall henceforth call it the rotational kinetic Sunyaev-Zeldovich effect (rk-SZE). To our knowledge, the rk-SZE was previously only considered by Cooray \& Chen (2002). In their work the rk-SZE was discussed for a gas

Send offprint requests to: J. Chluba, e-mail: jchluba@mpa-garching.mpg.de density profile following from hydrostatic equilibrium of the gas in the Navarro-Frenk-White dark matter density field within a halo (Navarro et al. 1996; Makino et al. 1998). Assuming isothermality this gas density profile is very well approximated by the commonly used isothermal $\beta$-model (Cavaliere \& Fusco-Femiano 1976), which is better applicable to analytical calculations. Here we focus on the contributions of the rk-SZE to the temperature fluctuations and the linear polarization of the $\mathrm{CMB}$ for the isothermal $\beta$-model finding a new method to measure the rotational properties of a cluster by performing multifrequency measurements of the position of the peak of the temperature fluctuation.

The paper is organized as follows. In Sect. 2 we state the model assumptions for the rotating cluster of galaxies and derive analytic formulae describing the rk-SZE. We also derive formulae describing combined thermal, kinetic and rotational kinetic SZE. In Sect. 3 we discuss our analytic results using recent observational data to estimate the effects for a set of 18 clusters in the redshift range from $z \sim 0.14$ to $z \sim 0.78$. Finally, we draw our conclusions in Sect. 4.

\section{Calculations}

\subsection{General equations}

\section{Kinetic Sunyaev-Zeldovich effect}

The relative change of intensity of the CMB due to the k-SZE of a small volume of electron gas with a line of sight optical depth $\mathrm{d} \tau=\sigma_{\mathrm{T}} n_{\mathrm{e}} \mathrm{d} l$, where $\sigma_{\mathrm{T}}$ is the Thomson cross section and $n_{\mathrm{e}}$ is the electron density, is given as (Sunyaev \& Zeldovich 1980)

$\mathrm{d}\left(\frac{\Delta I_{v}}{I_{v}}\right)=G_{v} \beta_{\|} \mathrm{d} \tau$ 
Here $\beta_{\|}$is the line of sight velocity in units of the speed of light $c$ and $G_{v}=x \mathrm{e}^{x} /\left(\mathrm{e}^{x}-1\right)$ with the dimensionless frequency $x=h v / k_{\mathrm{B}} T$, where $T$ is the temperature of the photon field, $h$ is the Planck constant and $k_{\mathrm{B}}$ is the Boltzmann constant. $\beta_{\|}$is defined to be positive if the cluster is approaching the observer. The degree of linear polarization of the CMB due to the k-SZE can be written as (Sunyaev \& Zeldovich 1980)

$\mathrm{d} P=\frac{1}{10} G_{v, \text { pol }} \beta_{\perp}^{2} \mathrm{~d} \tau$,

where now $\beta_{\perp}$ is the velocity component perpendicular to the line of sight and $G_{v, \text { pol }}$ is given as

$G_{v, \text { pol }}=\frac{\mathrm{e}^{x}\left(\mathrm{e}^{x}+1\right)}{2\left(\mathrm{e}^{x}-1\right)^{2}} x^{2}$.

The plane of polarization lies perpendicular to the direction of $\beta_{\perp}$. In the Rayleigh-Jeans part of the CMB spectrum $G_{v \text {,pol }}$ approaches unity and it increases as $\sim x^{2} / 2$ in the Wien tail (Sunyaev \& Zeldovich 1980; Sazonov \& Sunyaev 1999; Audit \& Simmons 1999).

\section{Thermal Sunyaev-Zeldovich effect}

The relative change of intensity of the CMB due to the th-SZE of a cluster of galaxies is given as (Zeldovich \& Sunyaev 1969)

$\frac{\Delta I_{v}}{I_{v}}=F_{v} \cdot y_{\mathrm{C}}$,

where $F_{v}$ and the Compton parameter $y_{\mathrm{C}}$ are defined as

$$
\begin{aligned}
& F_{v}=\frac{x \mathrm{e}^{x}}{\mathrm{e}^{x}-1}\left[x \frac{\mathrm{e}^{x}+1}{\mathrm{e}^{x}-1}-4\right], \\
& y_{\mathrm{C}}=\int \theta_{\mathrm{e}} \sigma_{\mathrm{T}} n_{\mathrm{e}} \mathrm{d} l
\end{aligned}
$$

with the dimensionless temperature of the electron gas, $\theta_{\mathrm{e}}=$ $k_{\mathrm{B}} T_{\mathrm{e}} / m_{\mathrm{e}} c^{2}$, where $m_{\mathrm{e}}$ is the rest mass of an electron. The typical temperature $T_{\mathrm{e}}$ of the electron gas inside clusters is $\sim 5 \mathrm{keV}$. Relativistic corrections thus lead to small changes in the form of $F_{v}$ which are neglected in the following (for details see Itoh et al. 1998; Challinor \& Lasenby 1998; Enßlin \& Kaiser 2000). In the Rayleigh-Jeans part of the CMB spectrum $F_{v}$ approaches -2 and it increases as $\sim x^{2}$ in the Wien tail. At the crossover frequency $x_{\mathrm{cr}} \sim 3.83$ the th-SZE vanishes and the $\mathrm{k}$-SZE reaches its maximum.

\subsection{Model of a rotating cluster of galaxies}

We describe the electron density profile of a rotating cluster of galaxies with an isothermal $\beta$-model (Cavaliere \& FuscoFemiano 1976). In order to account for asphericities, we introduce the parameter $\iota=r_{\mathrm{c}} / r_{z^{\prime}}$, where $r_{\mathrm{c}}$ is the core radius of the cluster and $r_{z^{\prime}}$ is the distance in the direction $z^{\prime}$, at which the electron density is equal to the density at the core radius in the spherical case. For $\iota<1$ the cluster is prolate, for $\iota>1$ it is oblate and spherical in the case $\iota=1$. This parameter mainly enters into the normalization of the optical depth and the shape of the cluster in the celestial plane. For illustrations we will adopt $\iota=1.1$. The modified isothermal $\beta$-model then, in the main axis system $S^{\prime}$, has the form

$n_{\mathrm{e}}(\boldsymbol{r})=n_{\mathrm{e} 0}\left(1+\frac{x^{\prime 2}+y^{\prime 2}+\iota^{2} z^{\prime 2}}{r_{\mathrm{c}}^{2}}\right)^{-\gamma}$,

where $n_{\mathrm{e} 0}$ is the central electron density and $\gamma=3 \beta / 2$, with a typical value $\beta=2 / 3$.

Very little, if anything, is known about the rotational properties of large-scale structures such as clusters of galaxies. But the existence of an angular momentum distribution in large scale structures is very likely: self-gravitating flows generally produce rotating structures on small scales (e.g. spiral galaxies, stars), even if the progenitor structure has a vanishing net angular momentum. Flattened geometries of clusters of galaxies may be a hint, that an angular momentum profile exists on larger scales as well. Recent numerical simulation of off-center cluster mergers predict, that a significant angular momentum associated with velocities up to few $10^{3} \mathrm{~km} \mathrm{~s}^{-1}$ can be induced (Roettiger \& Flores 2000; Roettiger et al. 1998). Also first observational evidence for an angular momentum distribution in clusters was found by measuring the Doppler shift of X-ray spectral lines (Dupke \& Bregman 2001). In recent simulations Bullock et al. (2001) found first evidence for an universal angular momentum profile in dark matter halos, which is consistent with solid body rotation with typical rotational velocity of $5 \%$ of the circular velocity. Assuming that the gas follows the dark matter motion, we therefore use solid body rotation to describe the velocity field of the ICM. (Further reasons are given in Cooray \& Chen 2002.)

\subsection{Kinetic Sunyaev-Zeldovich effect from cluster rotation}

Equation (1) can be used to calculate the relative intensity change of the CMB due to the internal motion of the cluster medium. If we assume that the angular velocity vector $\omega$ is anti-parallel to the $z^{\prime}$-axis and we define the frame $S$ of an observer such that the $z$-axis lies along the line of sight and the $x$-axis lies along the projection of $\omega$ onto the celestial plane, then the line of sight velocity is given as $\beta_{\|}=\beta_{\mathrm{c}} y S_{i} / r_{\mathrm{c}}$, where $\beta_{\mathrm{c}}=\omega r_{\mathrm{c}} / c$ is the rotational velocity at the core radius and $S_{i}$ denotes the sine of the inclination $i$ of the observer to the rotational axis. The derivation of the relative change of intensity for our simple model of a rotating cluster then reduces to the calculation of the optical depth as a function of $x$ and $y$, which is given in the Appendix A. It follows

$\left(\frac{\Delta I_{v}}{I_{v}}\right)_{\text {rot }}=G_{v} \beta_{\mathrm{c}} \frac{y S_{i}}{r_{\mathrm{c}}} \cdot \tau(x, y, i, \gamma)$,

where the definition of the optical depth $\tau(x, y, i, \gamma)$ is given in the Appendix (Eq. (A.2)). From Eq. (8) it is clear, that there is no effect for an inclination $i=0$ and it is maximal for $i=\pi / 2$.

In Fig. 1 the relative change of intensity due to the rk-SZE is shown for an oblate cluster $(\iota=1.1)$ with a cutoff radius $R=10 r_{\mathrm{c}}$, an inclination $i=\pi / 2$ and $\gamma=1.125$. The distinct dipolar-like pattern arises from the fact that one part of the gas is moving towards and the other part is moving away from the 


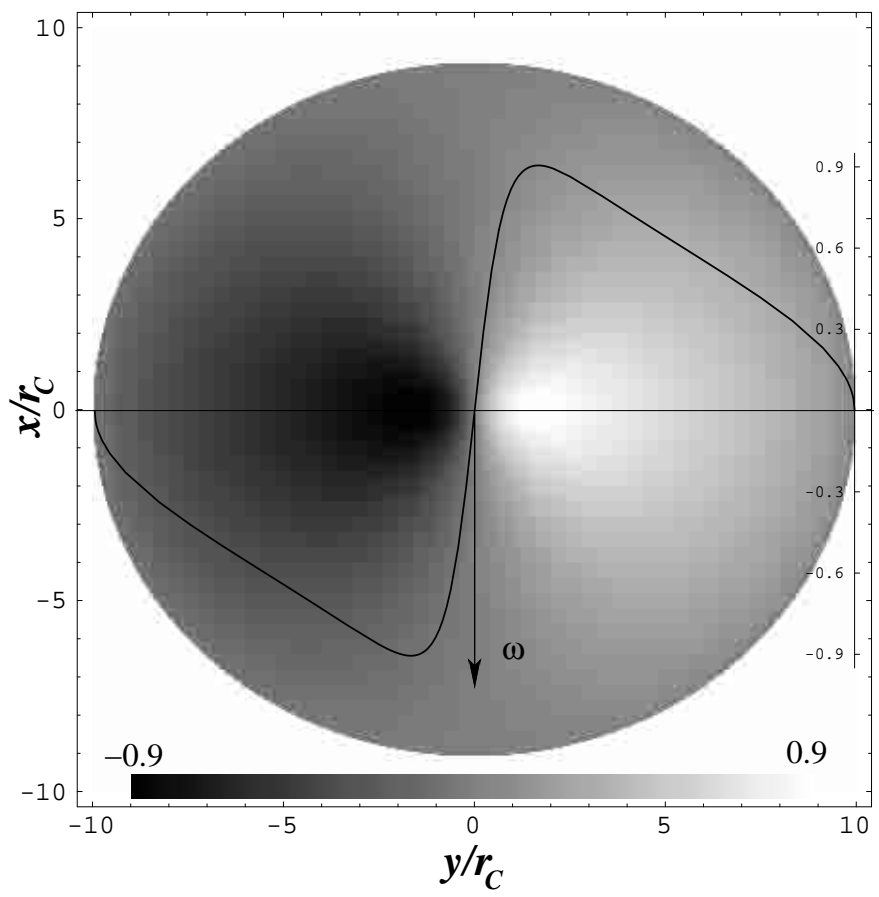

Fig. 1. Relative change of intensity (grayplot) in units of $G_{v} \beta_{\mathrm{c}} \tau_{\mathrm{c}}$ due to the rk-SZE for an oblate cluster $(\iota=1.1)$ with a cutoff radius $R=$ $10 r_{\mathrm{c}}$, an inclination $i=\pi / 2$ and $\gamma=1.125$. The overlayed plot (solid line) shows a cut along $x=0$.

observer. As can be seen in the overlayed plot, the peak value of the relative intensity change is about $\sim 0.9 \tau_{\mathrm{c}} \beta_{\mathrm{c}} G_{v}$. For further discussion see Sect. 3.

\subsection{Superposition of all the Sunyaev-Zeldovich contributions}

In general the relative change of intensity of the CMB has contributions from the thermal, the kinetic and the rotational kinetic SZE. Assuming that the cluster of galaxies is moving with a line of sight velocity $\beta_{\|, \mathrm{b}}$ one finds with Eqs. (1), (4) and (8)

$\frac{\Delta I_{v}}{I_{v}}=\left(F_{v} \theta_{\mathrm{e}}+G_{v}\left[\beta_{\|, \mathrm{b}}+\beta_{\mathrm{c}} \frac{y S_{i}}{r_{\mathrm{c}}}\right]\right) \cdot \tau(x, y, i, \gamma)$

for our simple model of a rotating cluster as described above. Depending on the observed frequency $v$ the importance of the different contributions varies. The th-SZE dominates in the Rayleigh-Jeans and Wien part of the CMB spectrum, whereas the k-SZE and the rk-SZE dominate around the crossover frequency $v_{\mathrm{c}} \sim 217 \mathrm{GHz}$. If the cluster is not rotating, the absolute value of relative change of intensity reaches its maximum at the center of the cluster, where the line of sight optical depth is highest. If there is only a contribution of the rk-SZE the relative change of intensity has two distinct extrema symmetrically around the center of the cluster.

It is possible to find the positions of the extrema of the relative change of intensity analytically from Eq. (9), if one assumes $R \rightarrow \infty$ and $\gamma>\frac{1}{2}$. For $\gamma \leq \frac{1}{2}$ the density profile decreases slower than $\sim 1 / r$ and therefore leads to infinite optical depth for $r \rightarrow \infty$. Although $R \rightarrow \infty$ implies superluminal rotation speeds far away from the center of the cluster, these unphysical contributions to the rk-SZE are small for typical values of $\gamma$ due to the rapidly, radially decreasing density (see Fig. 4 and the discussion).

Defining the parameter $\eta$ as

$\eta=\frac{2 G_{v} \beta_{\mathrm{c}}}{F_{v} \theta_{\mathrm{e}}+\beta_{\|, \mathrm{b}} G_{v}}$

and using Eqs. (9) and (A.5) one finds the formal solution

$y_{\mathrm{extr}}=-\frac{r_{\mathrm{c}}(2 \gamma-1)}{2 \eta S_{i}(\gamma-1)}\left[1 \pm \sqrt{1+\frac{2 \eta^{2} S_{i}^{2}(\gamma-1)}{(2 \gamma-1)^{2}}}\right]$

for the positions of the extrema relative to the center. Note that $y_{\text {extr }}$ is independent of $\iota$.

For $\gamma \rightarrow 1$ and $\eta \neq 2 G_{v} \beta_{\mathrm{c}}$ there is only one extremum at $\left|y_{\text {extr }}\right|=\left|\eta S_{i}\right| r_{\mathrm{c}} / 2$. In the case $\frac{1}{2}<\gamma<1$ the value of $\eta S_{i}$ is limited to the region $\left|\eta S_{i}\right| \leq \zeta$, where $\zeta$ is defined as

$\zeta=\frac{2 \gamma-1}{\sqrt{2|\gamma-1|}}$

For $\gamma \geq 1$ the value of $\eta S_{i}$ is arbitrary.

Equation (11) has the limiting forms $(\gamma \neq 1)$

$y_{\mathrm{extr}} \approx \mp \frac{\eta S_{i} r_{\mathrm{c}}}{2(2 \gamma-1)} \cdot\left[1+\frac{(1 \pm 1)(2 \gamma-1)^{2}}{\eta^{2} S_{i}^{2}(\gamma-1)}\right]$

for $\gamma>\frac{1}{2}$ and $\left|\eta S_{i}\right| \ll \zeta$ and

$y_{\mathrm{extr}} \approx \pm \frac{r_{\mathrm{c}}}{\sqrt{2(\gamma-1)}}$

for $\gamma>1$ and $\left|\eta S_{i}\right| \gg \zeta$. There are no extrema for $\gamma \leq 1$ and $\left|\eta S_{i}\right| \gg \zeta$.

The first case, i.e. Eq. (13), corresponds to a small contribution of the rk-SZE to the relative change of intensity compared to the thermal and the kinetic SZE. In this case there is an extremum at $y_{\text {extr }} \sim \eta S_{i} r_{\mathrm{c}} / 2(2 \gamma-1)$ near the center of the cluster and one far away from the center. The second case, i.e. Eq. (14), corresponds to a rk-SZE with negligible contributions of the thermal and the kinetic SZE. See Sect. 3 for further discussion.

\subsection{Polarization due to the rotational kinetic Sunyaev-Zeldovich effect}

Polarized radiation can be described by the Stokes parameters $I, Q, U, V$. The $Q$ and $U$ parameters describe the degree of linear polarization the $V$ parameter describes the degree of circular polarization (for further details see Rybicki \& Lightman 1979). We assume that the incident CMB radiation is isotropic and unpolarized $(Q=U=V=0)$ and that the scattering event can be treated as Thomson scattering (this means that the peculiar velocity $\beta_{\mathrm{p}}$ of the scattering electron obeys $\beta_{\mathrm{p}} \ll 1$ and $h v \ll m_{\mathrm{e}} c^{2}$. The latter is valid for CMB photons). Since Thomson scattering does not produce circular polarization we will drop the $V$-parameter in the following.

To calculate the contribution of the rk-SZE to the degree of linear polarization we have to find the scattered Stokes parameters $I^{\mathrm{s}}, Q^{\mathrm{s}}, U^{\mathrm{s}}$ in the rest frame of the observer. The degree of 
linear polarization is then given by

$P=\frac{\sqrt{\left(Q^{\mathrm{s}}\right)^{2}+\left(U^{\mathrm{s}}\right)^{2}}}{I^{\mathrm{tot}}}$

where the total intensity $I^{\text {tot }}$ is given as the sum of the incident intensity $I_{0}$, the scattered and the absorbed intensity: $I^{\text {tot }}=I_{0}(1-\tau)+I^{\mathrm{s}}$. The angle $\phi$ between the $x$-axis and the plane of polarization is given by

$\tan 2 \phi=\frac{U^{\mathrm{s}}}{Q^{\mathrm{s}}}$

Since the scattered Stokes parameters $Q^{\mathrm{s}}$ and $U^{\mathrm{s}}$ are proportional to $\tau$, consistent up to first order in $\tau$, the inverse of the total intensity in Eq. (15) can be approximated as $1 / I^{\text {tot }} \approx 1 / I_{0}$.

Assuming that the incident intensity $I_{0}$ is isotropic, the scattered Stokes parameters $\mathrm{d} Q^{\mathrm{s}}$ and $\mathrm{d} U^{\mathrm{s}}$ in the observer frame for a small volume of electron gas with optical depth $\mathrm{d} \tau$ and peculiar velocity $\beta_{\mathrm{p}}$ up to second order in $\beta_{\mathrm{p}}$ are given as (Sazonov \& Sunyaev 1999)

$\mathrm{d} Q^{\mathrm{s}}=\frac{1}{10} I_{0} G_{\nu, \mathrm{pol}} \beta_{\perp, \mathrm{p}}^{2} \cos (2 \chi) \mathrm{d} \tau$

$\mathrm{d} U^{\mathrm{s}}=-\frac{1}{10} I_{0} G_{\nu, \mathrm{pol}} \beta_{\perp, \mathrm{p}}^{2} \sin (2 \chi) \mathrm{d} \tau$,

where $\beta_{\perp, \mathrm{p}}$ is the velocity component in the celestial plane and $\chi$ is the angle between $\beta_{\perp, \mathrm{p}}$ and the $x$-axis of the observer.

For the model of a rotating cluster as described above the following relations can be found

$\beta_{\perp, \mathrm{r}}^{2} \cos \left(2 \chi_{\mathrm{r}}\right)=\beta_{\mathrm{c}}^{2}\left[\tilde{y}^{2} C_{i}^{2}-\left(\tilde{x} C_{i}+\tilde{z} S_{i}\right)^{2}\right]$

$\beta_{\perp, \mathrm{r}}^{2} \sin \left(2 \chi_{\mathrm{r}}\right)=-\beta_{\mathrm{c}}^{2} \tilde{y} C_{i}\left(\tilde{x} C_{i}+\tilde{z} S_{i}\right)$,

where $\beta_{\perp, \mathrm{r}}$ is the rotational velocity component in the celestial plane, $\chi_{\mathrm{r}}$ is the angle between $\beta_{\perp, \mathrm{r}}$ and the $x$-axis and $\tilde{x}$ and $\tilde{y}$ are the $x, y$-coordinates in units of the core radius $r_{\mathrm{c}}$. Inserting this into Eqs. (17a) and (17b) and integrating along the line of sight we find

$Q_{\mathrm{r}}^{\mathrm{s}}=\kappa \beta_{\mathrm{c}}^{2}\left[\left(\tilde{y}^{2}-\tilde{x}^{2}\right) C_{i}^{2} \cdot \tau-S_{i}^{2} \cdot J-2 \tilde{x} C_{i} S_{i} \cdot H\right]$

$U_{\mathrm{r}}^{\mathrm{s}}=2 \kappa \beta_{\mathrm{c}}^{2} \tilde{y} C_{i}\left[\tilde{x} C_{i} \cdot \tau+S_{i} \cdot H\right]$,

where $\kappa=\frac{1}{10} I_{0} G_{\gamma, \text { pol }}$ and the optical depth $\tau(x, y, i, \gamma)$ and integrals $J(x, y, i, \gamma)$ and $H(x, y, i, \gamma)$ are defined in the Appendix (Eqs. (A.2), (B.1a) and (B.1b)). Now, with Eq. (15) the degree of linear polarization due to the rk-SZE up to second order in $\beta_{\perp, \mathrm{r}}$ follows as

$$
\begin{gathered}
P_{\mathrm{r}}=\frac{1}{10} G_{v, \mathrm{pol}} \beta_{\mathrm{c}}^{2}\left[\left(\tilde{r}^{2} C_{i}^{2} \cdot \tau+S_{i}\left[S_{i} \cdot J+2 \tilde{x} C_{i} \cdot H\right]\right)^{2}\right. \\
\left.+4 \tilde{y}^{2} S_{i}^{2} C_{i}^{2} \cdot\left(H^{2}-\tau \cdot J\right)\right]^{\frac{1}{2}}
\end{gathered}
$$

Here $\tilde{r}$ is the distance from the center of the cluster in units of the core radius $r_{\mathrm{c}}$. The direction of the planes of polarization can be found with Eq. (16).
Here we note that with our simple model of a rotating cluster in general one cannot calculate the degree of polarization due to the rk-SZE assuming $R \rightarrow \infty$. For example, in the case $i=0$ the velocity component in the celestial plane at the center of the cluster is proportional to $\beta_{\perp} \propto \tilde{z}$. The electron density falls off as $n_{\mathrm{e}} \propto \tilde{z}^{-2 \gamma}$. Therefore the integral along the line of sight over $\beta_{\perp}^{2} n_{\mathrm{e}}$ diverges for $\gamma \leq 3 / 2$. Typical clusters have $\gamma \sim 1$ and hence have to be treated as finite systems. Alternatively one can modify the rotation model. The easiest way would be to assume a finite region rotating as a solid body followed by a region with constant rotation velocity, e.g. a rotation curve of spiral galaxies. This then removes the divergence and allows as to treat the case $R \rightarrow \infty$, but will not be discussed here any further.

For an inclination $i=0$ with Eq. (20) one finds

$P_{i=0, \mathrm{r}}=\frac{1}{10} G_{v, \mathrm{pol}} \beta_{\mathrm{c}}^{2} \tilde{r}^{2} \cdot \tau(x, y, 0, \gamma)$

for the degree of linear polarization. The planes of polarization lie along the radial vector. This can be also found by inserting $\beta_{\perp, i=0, \mathrm{r}}=\beta_{\mathrm{c}} \tilde{r}$ into Eq. (2) and integrating along the line of sight.

The degree of linear polarization due to the rk-SZE is shown in Fig. 2 for an oblate cluster $(\iota=1.1)$ with a cutoff radius $R=10 r_{\mathrm{c}}$ and $\gamma=1.125$. The short lines indicate the direction of the planes of polarization and their length the degree of polarization. As can be seen in the overlayed plot, the degree of polarization falls off steeply towards the edge of the cluster. This drop is related to the adopted cutoff radius: at the edges of a finite cluster the optical depth vanishes, implying a vanishing degree of polarization. In the case of an infinite cluster the optical depth decreases as $\tau \propto \tilde{r}^{1-2 \gamma}$ for $\tilde{r} \gg 1$. Therefore the product $\tilde{r}^{2} \tau$ diverges for $\gamma \leq 3 / 2$ at large distances from the center of the cluster, although in this case the results for $\tilde{r} \leq 1$ do not change significantly.

For an inclination $i=\pi / 2$ again with Eq. (20) one finds

$P_{i=\pi / 2, \mathrm{r}}=\frac{1}{10} G_{\nu, \mathrm{pol}} \beta_{\mathrm{c}}^{2} \cdot J(x, y, \pi / 2, \gamma)$

for the degree of linear polarization. The planes of polarization lie along the $x$-axis. Alternatively this result can be found by inserting $\beta_{\perp, i=\pi / 2, \mathrm{r}}=\beta_{\mathrm{c}} \tilde{z}$ into Eq. (2) and integrating along the line of sight.

\subsection{Polarization due to combined kinetic and rotational kinetic Sunyaev-Zeldovich effect}

If the cluster is rotating and in addition moving with the bulk translatory velocity $\beta_{\perp, \mathrm{b}}$ in the celestial plane one can find the relations

$$
\begin{aligned}
\beta_{\perp}^{2} \cos (2 \chi)= & \beta_{\perp, \mathrm{b}}^{2} \cos (2 \alpha)+\beta_{\perp, \mathrm{r}}^{2} \cos \left(2 \chi_{\mathrm{r}}\right) \\
& -2 \beta_{\perp, \mathrm{b}} \beta_{\mathrm{c}}\left[\left(\tilde{y} C_{\alpha}+\tilde{x} S_{\alpha}\right) C_{i}+\tilde{z} S_{\alpha} S_{i}\right] \\
\beta_{\perp}^{2} \sin (2 \chi)= & \beta_{\perp, \mathrm{b}}^{2} \sin (2 \alpha)+\beta_{\perp, \mathrm{r}}^{2} \sin \left(2 \chi_{\mathrm{r}}\right) \\
& -2 \beta_{\perp, \mathrm{b}} \beta_{\mathrm{c}}\left[\left(\tilde{y} S_{\alpha}-\tilde{x} C_{\alpha}\right) C_{i}-\tilde{z} C_{\alpha} S_{i}\right],
\end{aligned}
$$

where $\beta_{\perp}$ is the total velocity in the celestial plane, $\chi$ and $\alpha$ are the angles between the $x$-axis and $\beta_{\perp}$ and $\beta_{\perp, \mathrm{b}}$ respectively and 


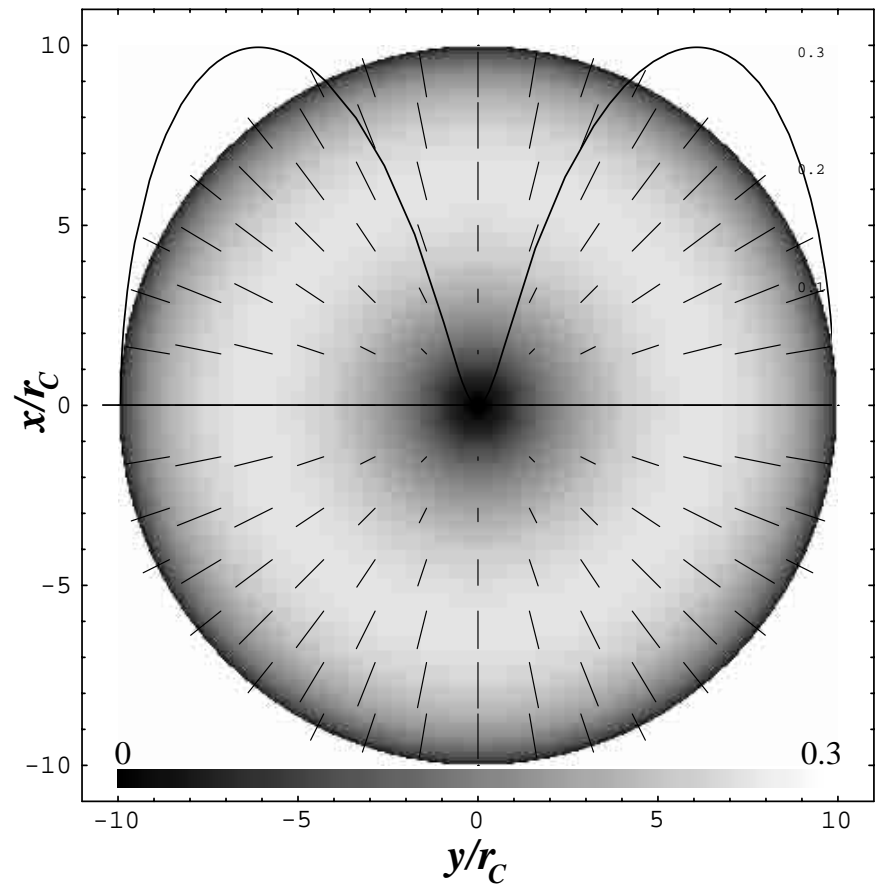

Fig. 2. Degree of linear polarization (grayplot) in units of $G_{v} \beta_{\mathrm{c}}^{2} \tau_{\mathrm{c}}$ for a rotating, oblate cluster $(\iota=1.1)$ with cutoff radius $R=10 r_{\mathrm{c}}$, an inclination $i=0$ and $\gamma=1.125$. The short lines indicate the direction of the planes of polarization and their length the degree of polarization. The overlayed plot (solid line) shows a cut along $x=0$.

the definitions (18) were used. Now with Eqs. (17a) and (17b) we find

$$
\begin{aligned}
Q^{\mathrm{s}}= & Q_{\mathrm{r}}^{\mathrm{s}}+\kappa \beta_{\perp, \mathrm{b}}^{2} \cos (2 \alpha) \cdot \tau \\
& -2 \kappa \beta_{\perp, \mathrm{b}} \beta_{\mathrm{c}}\left[\left(\tilde{y} C_{\alpha}+\tilde{x} S_{\alpha}\right) C_{i} \cdot \tau+S_{\alpha} S_{i} \cdot H\right] \\
U^{\mathrm{s}}= & U_{\mathrm{r}}^{\mathrm{s}}-\kappa \beta_{\perp, \mathrm{b}}^{2} \sin (2 \alpha) \cdot \tau \\
& +2 \kappa \beta_{\perp, \mathrm{b}} \beta_{\mathrm{c}}\left[\left(\tilde{y} S_{\alpha}-\tilde{x} C_{\alpha}\right) C_{i} \cdot \tau-C_{\alpha} S_{i} \cdot H\right] .
\end{aligned}
$$

To demonstrate the effects of the rk-SZE on the degree of linear polarization of the CMB, we now consider the two special cases $i=0$ and $i=\pi / 2$ with an additional bulk translatory motion of the cluster along the $y$-axis.

Case $i=0$ and $\alpha=\pi / 2$

With Eqs. (24) one finds in this case

$P_{i=0}=\frac{1}{10} G_{v, \mathrm{pol}} \cdot \beta_{\perp}^{2} \cdot \tau(x, y, 0, \gamma)$

with $\beta_{\perp}^{2}=\beta_{\perp, \mathrm{b}}^{2}+\beta_{\mathrm{c}}^{2} \tilde{r}^{2}+2 \beta_{\perp, \mathrm{b}} \beta_{\mathrm{c}} \tilde{x}$. The planes of polarization lie in the direction

$\boldsymbol{e}_{\mathrm{P}}=\frac{1}{\beta_{\perp}}\left(\begin{array}{c}\beta_{\perp, \mathrm{b}}+\beta_{\mathrm{c}} \tilde{x} \\ \beta_{\mathrm{c}} \tilde{y}\end{array}\right)$.

If the ICM is not rotating, the planes of polarization lie along the $x$-axis. With an additional rotation the planes are tilted by an angle

$\cos \varphi=\frac{\beta_{\perp, \mathrm{b}}+\beta_{\mathrm{c}} \tilde{x}}{\beta_{\perp}}$ which can be approximated by

$\cos \varphi \approx 1-\frac{1}{2} \tilde{y}^{2} \delta^{2}+\tilde{x} \tilde{y}^{2} \delta^{3}$

for small ratios $\delta=\beta_{\mathrm{c}} / \beta_{\perp, \mathrm{b}}$. This can be seen in Fig. 3 .

Case $i=\pi / 2$ and $\alpha=\pi / 2$

The total degree of linear polarization in this case is the sum of the contributions due to the rotation and due to the bulk translatory motion

$$
\begin{array}{r}
P_{i=\pi / 2}=\frac{1}{10} G_{\nu, \mathrm{pol}} \beta_{\perp, \mathrm{b}}^{2} \cdot \tau(x, y, \pi / 2, \gamma) \\
\cdot\left(1+\frac{\beta_{\mathrm{c}}^{2}}{\beta_{\perp, \mathrm{b}}^{2}} \frac{J(x, y, \pi / 2, \gamma)}{\tau(x, y, \pi / 2, \gamma)}\right)
\end{array}
$$

Assuming $\gamma=1$ the relative contribution due to the rk-SZE to the central degree of polarization is given as

$\frac{P_{i=\pi / 2, \mathrm{r}}}{P_{i=\pi / 2, \mathrm{~b}}}=\left[\frac{R}{\arctan R}-1\right] \cdot \delta^{2}$,

where $R$ is the cutoff radius of the cluster. For further discussion see the following section.

\section{Discussion}

\section{Kinetic Sunyaev-Zeldovich effect from cluster rotation}

In Fig. 1 the relative change of intensity of the CMB due to the rk-SZE is shown for an edge on $(i=\pi / 2)$ view of an oblate cluster $(\iota=1.1)$ with a cutoff radius $R=10 r_{\mathrm{c}}$ and $\gamma=1.125$ corresponding to $\beta=3 / 4$. As mentioned before the distinct dipolar-like pattern arises from the fact that one part of the gas is moving towards and the other part is moving away from the observer. If the cluster is viewed face on $(i=0)$ the effect is vanishing, since in that case there are no line of sight velocity components.

Figure 4 shows the dependence of the maximal relative change of intensity of the CMB due to the rk-SZE of $\gamma$ for different inclinations $i$. For increasing $\gamma$ the maximum value decreases. This is due to the fact that with the increasing steepness of the electron density profile the main contributions to the intensity change come from more inner regions of the cluster, which have smaller velocities. In the case $R \rightarrow \infty$ the relative change of intensity is only slightly bigger than for $R=10 r_{\mathrm{c}}$. This shows that the contributions of superluminal velocities far away from the center are negligible. There is no maximum for $\gamma \leq 1$ and $R \rightarrow \infty$. Figure 4 also shows the dependence of the position $y_{\max }$ of the maximal relative change of intensity of $\gamma$. For $R=10 r_{\mathrm{c}}$ it was found numerically with Eqs. (8). In the case $R \rightarrow \infty$ we used $y_{\max }=r_{\mathrm{c}} / \sqrt{2(\gamma-1)}$ from Eq. (14). For increasing $\gamma$ the position of the maximum moves towards the center of the cluster. This is again due to the increasing steepness of the electron density profile. The position of the maximum does not depend on the inclination $i$ and the parameter $\iota$. 


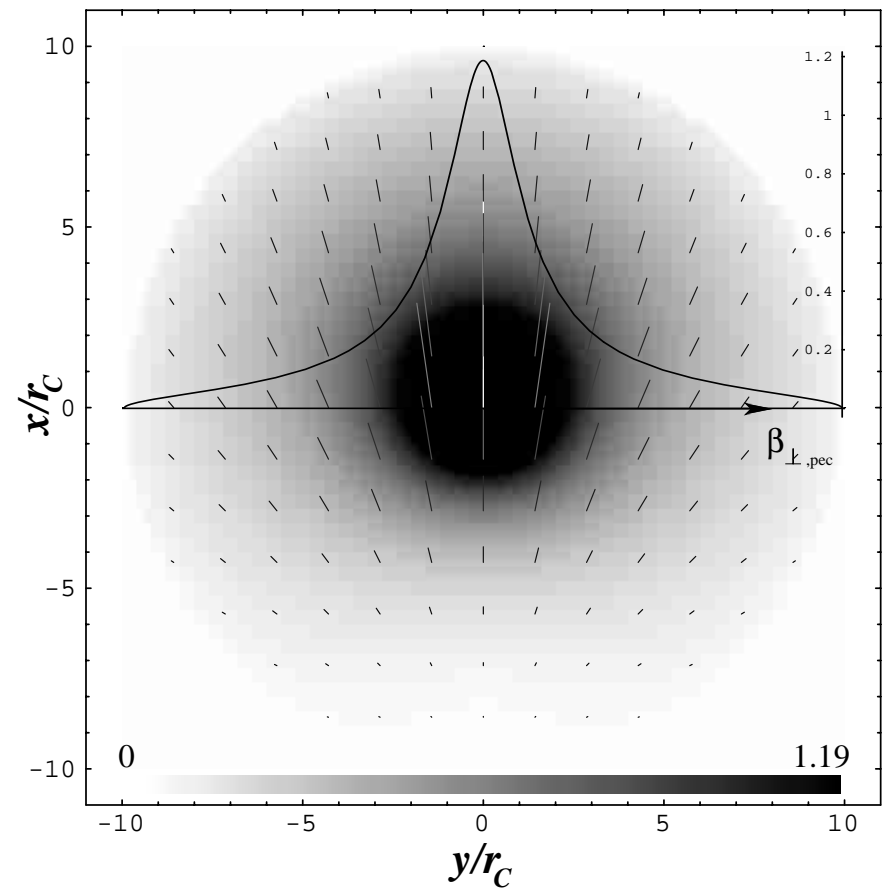

Fig. 3. Degree of linear polarization (grayplot; note that we used a scaling convention opposite to Fig. 2) in units of $G_{v} \beta_{\mathrm{c}}^{2} \tau_{\mathrm{c}}$ for a rotating, oblate cluster $(\iota=1.1)$ with cutoff radius $R=10 r_{\mathrm{c}}$, an inclination $i=0$ and $\gamma=1.125$ which is in addition moving with $\beta_{\perp, \mathrm{b}}=10 \beta_{\mathrm{c}}$ in $y$-direction. The short lines indicate the direction of the planes of polarization and their length the degree of polarization. The overlayed plot (solid line) shows a cut along $x=0$.

\section{Estimates from observational data}

In a recent paper of Reese et al. (2002) the th-SZE at a frequency of $v \sim 30 \mathrm{GHz}$ for a sample of 18 clusters in the redshift range from $z \sim 0.14$ to $z \sim 0.78$ was examined. If we assume that all the clusters in the sample are rotating and they are all observed edge-on we can estimate the position of the maximal change of intensity due to the rk-SZE relative to the center of the cluster with the results for $\beta$ and $r_{\mathrm{c}}$ given in Reese et al. (2002). The estimates are given in Table 1 . In the analysis of Reese et al. (2002) the usual assumption of an infinite cluster $R \rightarrow \infty$ was made. In this case, with our model of a rotating cluster there are no distinct extrema for $\gamma \leq 1$ (see (14)). The estimates for the case $R=10 r_{\mathrm{c}}$ correspond to the assumption of an infinite cluster but a finite region of rotation. A typical value for the position of the maximal relative change of intensity relative to the center of the cluster for this sample is $y_{\max } \sim 2.6 r_{\mathrm{c}}$.

With the results of Reese et al. (2002) it is also possible to estimate the central optical depth $\tau_{\mathrm{c}}$. As mentioned in their paper, relativistic corrections to the th-SZE for their set of clusters are typically of the order of a few $\%$ in the observed frequency band. Neglecting these corrections we can make use of Eqs. (5) and (6). Assuming an infinite isothermal $\beta$-model (Cavaliere \& Fusco-Femiano 1976) for the electron density one finds

$\tau_{\mathrm{c}}=\frac{2 \Gamma(\gamma)}{\sqrt{\pi} \Gamma\left(\gamma-\frac{1}{2}\right)} \frac{1}{F_{\gamma} \theta_{\mathrm{e}}} \frac{\Delta T_{0}}{T_{\mathrm{CMB}}}$

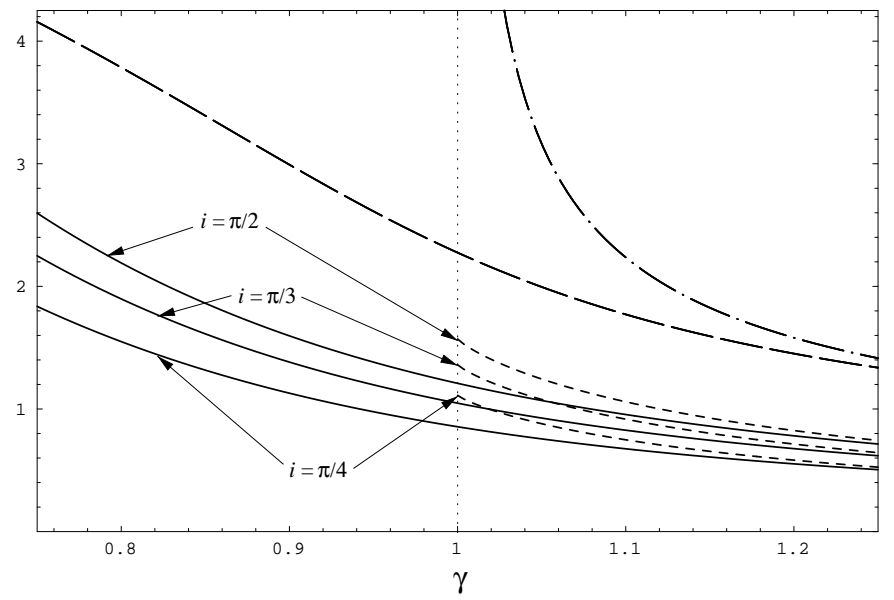

Fig. 4. Maximal relative change of intensity $\left(\Delta I_{v} / I_{v}\right)_{\text {rot }}$ of a spherical cluster in units of $\tau_{\mathrm{c}} \beta_{\mathrm{c}} G_{v}$ for different inclinations $i$ : the solid lines correspond to the case $R=10 r_{\mathrm{c}}$ and the short-dashed lines to $R=\infty$. Also the position $y_{\max }$ of the maximal relative change of intensity in units of the core radius $r_{\mathrm{c}}$ is shown for the case $R=10 r_{\mathrm{c}}$ (long-dashed) and $R=\infty$ (dashed-dotted). The value of $y_{\max }$ does not depend on the inclination $i$ and the parameter $\iota$.

for the central optical depth, where $\Gamma(x)$ is the Gamma-function and $\Delta T_{0} / T_{\mathrm{CMB}}$ is the central temperature decrement due to the th-SZE relative to the CMB temperature $T_{\mathrm{CMB}}=2.728 \mathrm{~K}$ (Fixsen et al. 1996). The typical central optical depth for the sample of clusters of Reese et al. (2002) is $\tau_{\mathrm{c}} \sim 10^{-3}-10^{-2}$. With the estimates of the optical depth for each cluster it is possible to calculate the value of the maximal relative change of intensity due to the rk-SZE. The results are given in Table 1. As mentioned before, the results for $R \rightarrow \infty$ and $R=10 r_{\mathrm{c}}$ agree well with each other. For the maximum of $\left(\Delta I_{v} / I_{v}\right)_{\text {rot }}$ we find a typical value of $\sim 1.4 G_{v} \beta_{\mathrm{c}} \tau_{\mathrm{c}}$.

Cooray \& Chen (2002) argue that the velocity at the core radius can be of the order of a few up to a few ten $\mathrm{km} \mathrm{s}^{-1}$, that is $\beta_{\mathrm{c}} \sim 10^{-5}-10^{-4}$, if there was a recent merger maybe even $\beta_{\mathrm{c}} \sim 5 \times 10^{-4}$ (Roettiger \& Flores 2000; Roettiger et al. 1998). For an observation near the crossover frequency, $v_{\mathrm{c}} \sim 217 \mathrm{GHz}$, where the kinetic and the rotational kinetic SZE reach their maximum, the relative change of intensity of the CMB due to the rk-SZE should lead to a temperature change of the order of $\Delta T_{\text {rot }} \sim 10^{-1}-10 \mu \mathrm{K}$, for a recent merger even up to $\Delta T_{\text {rot }} \sim 100 \mu \mathrm{K}$. For the sample of clusters from Reese et al. (2002) the expected temperature change at the crossover frequency is given in Table 1 assuming $\beta_{\mathrm{c}} \sim 10^{-4}$. It ranges from $\Delta T_{\text {rot }} \sim 3.5 \mu \mathrm{K}$ for $\beta_{\mathrm{c}} \sim 10^{-4}$ up to $\Delta T_{\text {rot }} \sim 146 \mu \mathrm{K}$ for a recent merger. Our results lie in the range predicted by Cooray \& Chen (2002).

\section{Superposition of all the Sunyaev-Zeldovich contributions}

If the SZE of a rotating cluster is observed, there are in general contributions of all the different SZEs. The thermal and the kinetic SZE both peak in the center of the cluster, while the rk-SZE has a maximum and a minimum displaced symmetrically around the center. We have shown that the absolute 
Table 1. Estimates for $y_{\max }$ relative to the center of the cluster, $\left(\Delta I_{v} / I_{v}\right)_{\text {rot }}$ and the peak temperature change $\Delta T_{\text {rot }}$ at the crossover frequency for a sample of 18 clusters from Reese et al. (2002) assuming solid body rotation of the ICM and an inclination $i=\pi / 2$.

\begin{tabular}{|c|c|c|c|c|c|}
\hline \multirow[b]{2}{*}{ Cluster } & \multicolumn{2}{|c|}{$\begin{array}{c}y_{\max } \\
{[\operatorname{arcsec}]}\end{array}$} & \multicolumn{2}{|c|}{$\begin{array}{c}\left(\Delta I_{v} / I_{v}\right)_{\mathrm{rot}} \\
{\left[G_{v} \beta_{\mathrm{c}} \times 10^{-3}\right]} \\
\end{array}$} & \multirow{2}{*}{$\begin{array}{c}\Delta T_{\text {rot }} \\
{\left[\frac{\beta_{\mathrm{c}}}{10^{-4}} \mu \mathrm{K}\right]} \\
R=10 r_{\mathrm{c}}\end{array}$} \\
\hline & $R=10 r_{\mathrm{c}}$ & $R=\infty$ & $R=10 r_{\mathrm{c}}$ & $R=\infty$ & \\
\hline MS 1137 & 29.2 & 32.4 & 6.8 & 7.2 & 7.3 \\
\hline MS 0451 & 49.6 & 53.7 & 6.3 & 6.7 & 6.8 \\
\hline CL 0016 & 71.2 & 85.1 & 8.2 & 9.0 & 8.8 \\
\hline R1347 & 26.5 & - & 27.3 & - & 29.1 \\
\hline A 370 & 156.4 & - & 13.8 & - & 14.8 \\
\hline MS 1358 & 49.8 & - & 6.5 & - & 7.0 \\
\hline A1995 & 61.4 & 69.9 & 5.8 & 6.2 & 6.2 \\
\hline A611 & 59.7 & - & 8.9 & - & 9.5 \\
\hline A697 & 140.2 & - & 10.2 & - & 10.9 \\
\hline A1835 & 37.2 & - & 19.9 & - & 21.2 \\
\hline A2261 & 62.5 & - & 14.0 & - & 15.0 \\
\hline A773 & 136.3 & - & 8.8 & - & 9.4 \\
\hline A 2163 & 193.2 & 589.9 & 8.8 & 11.0 & 9.4 \\
\hline A520 & 160.9 & 169.0 & 3.5 & 3.6 & 3.7 \\
\hline A1689 & 76.8 & - & 11.4 & - & 12.2 \\
\hline A665 & 202.0 & - & 5.1 & - & 5.4 \\
\hline A2218 & 138.9 & 244.8 & 5.7 & 6.7 & 6.1 \\
\hline A1413 & 121.7 & - & 6.8 & - & 7.3 \\
\hline
\end{tabular}

value of the relative change of intensity for the superposition of all the different SZEs has a maximum near the center. For an infinite cluster its position is approximately at $y_{\text {extr }}=$ $\eta S_{i} r_{\mathrm{c}} / 2(2 \gamma-1)$ for small $\eta$, where $\eta$ gives the relative contribution of the rk-SZE to the sum of the thermal and the kinetic SZE and is strongly dependent on the frequency (see Eq. (10)). Note that $y_{\text {extr }}$ is independent of $\iota$.

Observing a cluster at the crossover frequency the parameter $\eta$ becomes equal to $2 \beta_{\mathrm{c}} / \beta_{\|, \mathrm{b}}$ and is therefore for typical bulk translatory velocities $\beta_{\|, \mathrm{b}} \sim 10^{-4}-10^{-3}$ of the order $\eta \sim 2 \times 10^{-2}-2 \times 10^{-1}$. Assuming an inclination $i=\pi / 2$ the displacement of the maximal relative change of intensity is a few $\%$ of the core radius of the cluster. For a typical core radius of a few ten arcsec this means a displacement up to a few arcsec. The displacement is maximal at the crossover frequency and is negligible in the Rayleigh-Jeans part of the CMB spectrum. Since the main contribution to the peak value of the relative change of intensity in the considered case comes from the $\mathrm{k}$-SZE, one can in principle use this effect to measure the rotational velocity component of clusters by measuring the frequency dependent displacement.

\section{Polarization due to the kinetic Sunyaev-Zeldovich effect from cluster rotation}

The degree of linear polarization of the $\mathrm{CMB}$ radiation due to the rk-SZE for arbitrary inclination of the observer to the rotation axis can be described with Eq. (20). For the case $i=0$ we showed, that the planes of polarization for a superposed kinetic and rotational kinetic SZE are tilted by a angle given by Eq. (27). If we assume that the bulk translatory velocity $\beta_{\perp, \mathrm{b}}$ is 10 to 100 times larger than the rotational velocity at the core radius $\beta_{\mathrm{c}}$, then the planes of polarization at the cluster core radius are tilted by an angle of the order of $\sim 0.6^{\circ}$ to $\sim 6^{\circ}$ (see Eq. (28)). This tilt is frequency independent and can therefore be distinguished from other effects leading to rotation of the planes of polarization, like Faraday rotation due to intracluster magnetic fields.

For a rotating cluster with cutoff radius $R=10 r_{\mathrm{c}}$ and $\gamma=1$, which is in addition moving with a bulk translatory velocity $\beta_{\perp, \mathrm{b}}=10 \beta_{\mathrm{c}}$, the contribution of the rk-SZE to the central degree of polarization is of the order of $\sim 6 \%$ for an edge-on observation (see Eq. (30)). This is equivalent to a temperature change of the order of $\Delta T_{\mathrm{r}} \sim 10^{-4} \mu \mathrm{K}$ and therefore still a challenge for the future. For high precision measurement of the transversal peculiar velocity component $\beta_{\perp \text {,pec }}$ of clusters the rk-SZE has to be taken into account.

Here we want to note that the most important contribution to the polarization $(\sim 0.1(\tau / 0.02) \mu \mathrm{K})$ arises from the quadrupole component of the CMB anisotropy (Sazonov \& Sunyaev 1999). Another important contribution to the polarization $\left(\sim 0.01 \theta_{\mathrm{e}} \tau^{2}\right)$, which can overcome the k-SZE and the rk-SZE at high frequencies, comes from multiple scattering inside rich clusters (for a detailed overview see Sazonov \& Sunyaev 1999). Since the frequency dependence of all these effects is different they can be separated by multifrequency measurements.

\section{Conclusions}

We have derived analytic formulae describing the relative change of intensity and the degree of linear polarization of the CMB radiation due to the kinetic SZE of a rotating cluster of galaxies (Eqs. (8), (20)). We also have found analytic formulae for the superposition of all possible SZE contributions (Eqs. (9), (24)). We have estimated the possible amplitude of 
the relative change of intensity due to the rk-SZE for a sample of 18 clusters of galaxies in a redshift range from $z \sim 0.14$ to $z \sim 0.78$ (see Table 1). Our results show, that the contribution of the rk-SZE to the peak temperature change for this sample can be expected to range from $\Delta T_{\text {rot }} \sim 3.5 \mu \mathrm{K}$ for $\beta_{\mathrm{c}} \sim 10^{-4}$ up to $\Delta T_{\text {rot }} \sim 146 \mu \mathrm{K}$ for a recent merger $\left(\beta_{\mathrm{c}} \sim 5 \times 10^{-4}\right)$ of rich clusters. This agrees with the range predicted by Cooray \& Chen (2002).

We have also shown that due to the superposition of the thermal, the kinetic and the rotational kinetic SZE there is a frequency dependent displacement (Eq. (11)) of the peak value of the relative intensity change, which in principle can be used to examine the properties of the rotational velocity component of the ICM. Ground based interferometric telescopes should be able to detect this effect in the near future. Since the angular momentum distribution is not easily measurable with other observational techniques, this aspect of the rk-SZE might provide a new possibility of getting insights into the internal dynamics of clusters of galaxies.

In the future polarization measurements of the CMB radiation may become feasible. In this work we have shown that the polarization map following from the k-SZE can alter significantly due to the rk-SZE. Although the contribution of the rk-SZE to the degree of polarization relative to the $\mathrm{k}-\mathrm{SZE}$ is only of the order of a few $\%$ (quantitatively $\Delta T_{\mathrm{r}} \sim 10^{-4} \mu \mathrm{K}$ ), the directions of planes of polarization can be tilted by an angle, which is of the order of a few degrees (Eq. (28)). Since this tilt is frequency independent it can be easily separated from other effects, for example Faraday rotation. In the Wien region of the CMB spectrum the degree of polarization due to the kinetic and the rotational kinetic SZE increases by a factor of 10-100. Therefore measurements of the CMB polarization should be performed in this frequency range.

Acknowledgements. We acknowledge hospitality at the UniversitätsSternwarte Göttingen, where most of this work was done in the summer of 2001 as part of the diploma thesis of J. C. We also want to thank S. Y. Sazonov and T. A. Enßlin for valuable discussions which helped improving the paper.

\section{Appendix A: Derivation of the optical depth}

The optical depth is defined as the line of sight integral $\tau=$ $\int \sigma_{\mathrm{T}} n_{\mathrm{e}} \mathrm{d} z$ over the electron density. Transforming the electron density profile (7) into the frame of the observer and defining the following abbreviations

$$
\begin{aligned}
\xi & =b z-\frac{d}{b} \\
A & =\sqrt{1+a^{2}-\left(\frac{d}{b}\right)^{2}} \\
a^{2} & =\frac{\left(C_{i}^{2}+\iota^{2} S_{i}^{2}\right) x^{2}+y^{2}}{r_{\mathrm{c}}^{2}} \\
b^{2} & =S_{i}^{2}+\iota^{2} C_{i}^{2} \\
d & =\frac{C_{i} S_{i}\left(\iota^{2}-1\right) x}{r_{\mathrm{c}}},
\end{aligned}
$$

where we used $S_{i}=\sin i$ and $C_{i}=\cos i$, the optical depth as a function of $x$ and $y$ is given as

$\tau(x, y, i, \gamma)=\frac{\tau_{\mathrm{c}}}{\sqrt{S_{i}^{2}+\iota^{2} C_{i}^{2}}} \cdot I(x, y, i, \gamma)$.

Here we defined

$$
\tau_{\mathrm{c}}=2 \sigma_{\mathrm{T}} n_{\mathrm{e} 0} r_{\mathrm{c}}
$$

$$
\begin{aligned}
I(x, y, i, \gamma) & =\int_{0}^{\xi_{\max }}\left(A^{2}+\xi^{2}\right)^{-\gamma} \mathrm{d} \xi \\
\xi_{\max } & =\sqrt{\frac{R^{2}}{r_{\mathrm{c}}^{2}}+1-A^{2}} .
\end{aligned}
$$

The upper limit $\xi_{\max }$ results from the boundary of the cluster, which is parametrized by $R$ assuming an ellipsoidal boundary surface. The integral (A.3b) can be evaluated analytically and is given as

$I(x, y, i, \gamma)=\frac{\xi_{\max }}{A^{2 \gamma}} \cdot{ }_{2} F_{1}\left(\frac{1}{2}, \gamma ; \frac{3}{2} ;-\frac{\xi_{\max }^{2}}{A^{2}}\right)$,

where we made use of the hypergeometric function ${ }_{2} F_{1}(a, b ; c ; z)$ (see Abramovitz \& Stegun 1965).

In the case when $R \rightarrow \infty$ and for $\gamma>1 / 2$ the integral $I(x, y, i, \gamma)$ reduces to

$I_{\infty}(x, y, i, \gamma)=\frac{\sqrt{\pi}}{2} \frac{\Gamma\left(\gamma-\frac{1}{2}\right)}{\Gamma(\gamma)}\left(A^{2}\right)^{\frac{1}{2}-\gamma}$,

where $\Gamma(z)=\int_{0}^{\infty} t^{z-1} \mathrm{e}^{-t} \mathrm{~d} t$ is the Gamma-function.

In the case of $\gamma=1$ the integral $I(x, y, i, \gamma)$ reduces to

$I(x, y, i, 1)=\frac{1}{A} \arctan \left(\frac{\xi_{\max }}{A}\right)$

and has the limiting form $I_{\infty}(x, y, i, 1)=\pi / 2 A$ for $R \rightarrow \infty$.

\section{Appendix B: Definition of the integrals $J(x, y, i, \gamma)$ and $H(x, y, i, \gamma)$}

In the derivation of the degree of linear polarization due to the rotation of the cluster medium the integrals

$$
\begin{aligned}
J(x, y, i, \gamma) & =\frac{\tau_{\mathrm{c}}}{b^{3}} \cdot I_{2}(x, y, i, \gamma) \\
H(x, y, i, \gamma) & =\frac{\tau_{\mathrm{c}}}{b^{2}} \cdot I_{1}(x, y, i, \gamma)
\end{aligned}
$$

arise, where we used the abbreviations of Appendix A and defined the integral $I_{n}$ as

$I_{n}(x, y, i, \gamma)=\frac{1}{2} \int_{-\xi_{\max }}^{\xi_{\max }}\left(\xi+\frac{d}{b}\right)^{n}\left(A^{2}+\xi^{2}\right)^{-\gamma} \mathrm{d} \xi$.

In the limit $R \rightarrow \infty$ the integral $I_{n}(x, y, i, \gamma)$ diverges for the combinations $n=2, \gamma \leq 3 / 2$ and $n=1, \gamma \leq 1$. For finite $R$ the solutions

$$
\begin{aligned}
I_{1}(x, y, i, \gamma)= & \frac{t \xi_{\max }}{A^{2 \gamma}} \cdot{ }_{2} F_{1}\left(\frac{1}{2}, \gamma ; \frac{3}{2} ;-\frac{\xi_{\max }^{2}}{A^{2}}\right) \\
I_{2}(x, y, i, \gamma)= & \frac{1}{3} \frac{\xi_{\max }^{3}}{A^{2 \gamma}} \cdot{ }_{2} F_{1}\left(\frac{3}{2}, \gamma ; \frac{5}{2} ;-\frac{\xi_{\max }^{2}}{A^{2}}\right) \\
& +\frac{t^{2} \xi_{\max }}{A^{2 \gamma}} \cdot{ }_{2} F_{1}\left(\frac{1}{2}, \gamma ; \frac{3}{2} ;-\frac{\xi_{\max }^{2}}{A^{2}}\right)
\end{aligned}
$$


can be found, where we defined $t=d / b$ and made use of the hypergeometric function ${ }_{2} F_{1}(a, b ; c ; z)$ (see Abramovitz \& Stegun 1965). For $\gamma=1$ and finite $R$ one finds the solutions

$$
\begin{aligned}
& I_{1}(x, y, i, 1)=\frac{t}{A} \cdot \arctan \left(\frac{\xi_{\max }}{A}\right) \\
& I_{2}(x, y, i, 1)=\xi_{\max }-\frac{A^{2}-t^{2}}{A} \cdot \arctan \left(\frac{\xi_{\max }}{A}\right) .
\end{aligned}
$$

\section{References}

Abramovitz, M., \& Stegun, I. A. 1965 (Dover Publications, Inc., New York)

Audit, E., \& Simmons, F. L. 1999, MNRAS, 305, L27

Birkinshaw, M. 1999, Phys. Rep., 310, 97

Bullock, J. S., Dekel, A., Kolatt, T. S., et al. 2001, ApJ, 555, 240

Challinor, A., \& Lasenby, A. 1998, ApJ, 499, 1

Cavaliere, A., \& Fusco-Femiano, R. 1976, A\&A, 49, 137

Cooray, A., \& Chen, X. 2002, ApJ, 573, 43
Dupke, R. A., \& Bregman, J. N. 2001, ApJ, 562, 266

Itoh, N., Kohyama, Y., \& Nozawa, S. 1998, ApJ, 502, 7

Enßlin, T. A., \& Kaiser, C. R. 2000, A\&A, 360, 417

Fixsen, D. J.,Cheng, E. S., Gales, J. M., et al. 1996, ApJ, 473, 576

Korolev, V. A., Syunyaev, R. A., \& Yakubtsev, L. A. 1986, Sov. Astr. Lett, 3,141

Kneissl, R., Jones, M. E., Saunders, R., et al. 2001, MNRAS, 328, 783

Navarro, J. F., Frenk, C. S., \& White, S. D. M. 1996, ApJ, 462, 563

Makino, N., Sasaki, S., \& Suto, Y. 1998, ApJ, 497, 555

Reese, E. D., Carlstrom, J. E., Joy, M., et al. 2002, preprint [astro-ph/0205350]

Roettiger, K., \& Flores, R. 2000, ApJ, 538, 92

Roettiger, K., Stone, J. M., \& Mushotzky, R. F. 1998, ApJ, 493, 62

Rybicki, G. B., \& Lightman, A. P. 1979, Radiative Processes in Astrophysics (Wiley Interscience Publication)

Sazonov, S. Y., \& Sunyaev, R. A. 1999, MNRAS, 310, 765

Sunyaev, R. A., \& Zeldovich, Ya. B. 1980, MNRAS, 190, 413

White, M., \& Cohn, J. D. 2002, preprint [astro-ph/0203120]

Zeldovich, Ya. B., \& Sunyaev, R. A. 1969, Ap\&SS, 4, 301 